\title{
Measurements on NASA Langley Durable Combustor Rig by TDLAT: Preliminary Results
}

\author{
Kristin M. Busa ${ }^{1}$, Erik N. Ellison ${ }^{2}$, Brian J. McGovern ${ }^{3}$, and James C. McDaniel ${ }^{4}$ \\ University of Virginia, Charlottesville, VA, 22904 \\ and \\ Glenn S. Diskin ${ }^{5}$, Maxwell J. DePiro ${ }^{6}$, Diego P. Capriotti ${ }^{7}$, and Richard L. Gaffney ${ }^{8}$ \\ NASA Langley Research Center, Hampton, VA, 23681
}

\begin{abstract}
Detailed knowledge of the internal structure of high-enthalpy flows can provide valuable insight to the performance of scramjet combustors. Tunable Diode Laser Absorption Spectroscopy (TDLAS) is often employed to measure temperature and species concentration. However, TDLAS is a path-integrated line-of-sight (LOS) measurement, and thus does not produce spatially resolved distributions. Tunable Diode Laser Absorption Tomography (TDLAT) is a non-intrusive measurement technique for determining two-dimensional spatially resolved distributions of temperature and species concentration in high enthalpy flows. TDLAT combines TDLAS with tomographic image reconstruction. Several separate line-of-sight TDLAS measurements are analyzed in order to produce highly resolved temperature and species concentration distributions. Measurements have been collected at the University of Virginia's Supersonic Combustion Facility (UVaSCF) as well as at the NASA Langley Direct-Connect Supersonic Combustion Test Facility (DCSCTF). Measurements collected at the DCSCTF required significant modifications to system hardware and software designs due to its larger measurement area and shorter test duration. Initial LOS measurements from the NASA Langley DCSCTF operating at an equivalence ratio of 0.5 are presented. Results show the capability of TDLAT to adapt to several experimental setups and test parameters.
\end{abstract}

\section{Nomenclature}

$\begin{array}{ll}\eta_{c} & =\text { combustion efficiency } \\ n_{H 2 O} & =\text { water number density distribution } \\ V_{x}(y, z) & =\text { axial velocity distribution } \\ \dot{N}_{\text {Hydrogen,injected }} & =\text { total injected hydrogen flux }\end{array}$

${ }^{1}$ Graduate Research Assistant, Department of Mechanical and Aerospace Engineering Charlottesville, VA 22904 AIAA Student Member.

${ }^{2}$ Undergraduate Research Assistant, Department of Mechanical and Aerospace Engineering Charlottesville, VA 22904 AIAA Student Member.

${ }^{3}$ Undergraduate Research Assistant, Department of Mechanical and Aerospace Engineering Charlottesville, VA 22904 AIAA Student Member.

${ }^{4}$ Professor, Department of Mechanical and Aerospace Engineering, Charlottesville, VA 22904 AIAA Associate Fellow.

5 Senior Research Scientist, Chemistry and Dynamics Branch, Mail Stop 483, NASA Langley Research Center Hampton, VA 23681, AIAA Senior Member.

${ }^{6}$ Graduate Co-op Engineer, Hypersonic Airbreathing Propulsion Branch, Mail Stop 168, NASA Langley Research Center, Hampton, VA 23681, AIAA Student Member.

${ }^{7}$ Research Engineer, Hypersonic Airbreathing Propulsion Branch, Mail Stop 168, NASA Langley Research Center, Hampton, VA 23681.

8 Aerospace Engineer, Hypersonic Airbreathing Propulsion Branch, Mail Stop 168, NASA Langley Research Center, Hampton, VA 23681, AIAA Senior Member. 


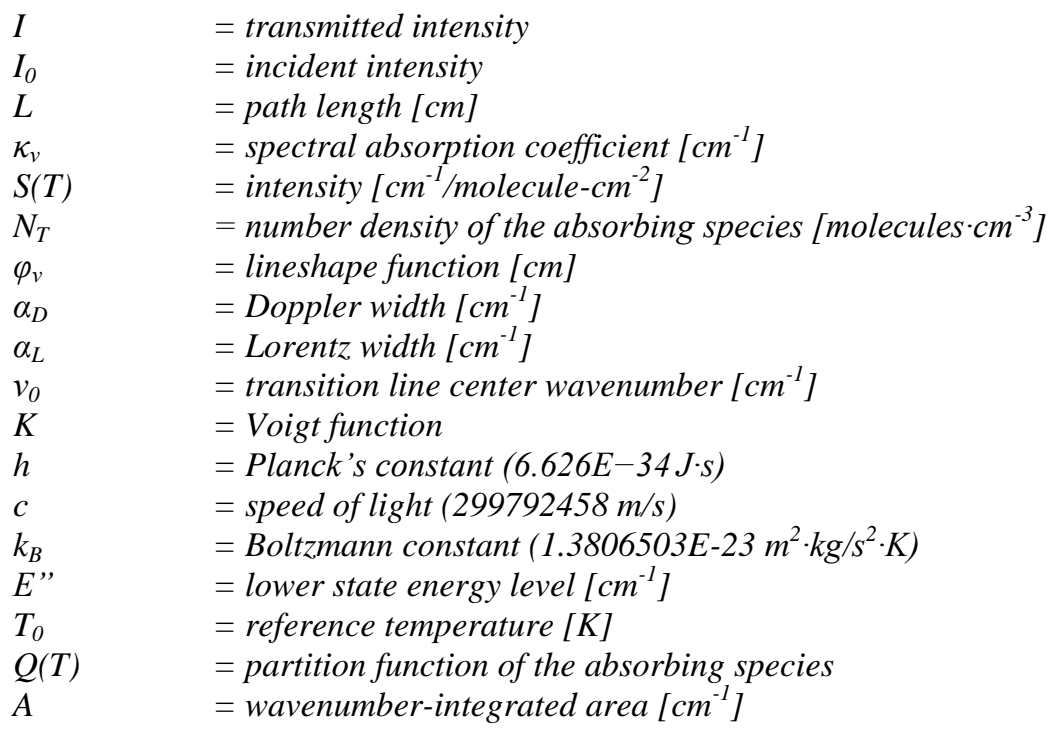

\section{Introduction}

$\mathrm{T}$

HE use of diode lasers for measuring gas properties originated in the 1970's following the demonstration of direct current injection semiconductor lasers ${ }^{1}$. In 1977, one of the earliest demonstrations of using tunable IR diode lasers to non-intrusively acquire in situ measurement of gas properties in combusting gases was by Hanson $e t$ $a l^{2}$. The field of diode laser absorption sensing has grown rapidly since and has been applied in laboratory and industrial flows ${ }^{1}$. Tunable Diode Laser Absorption Spectroscopy (TDLAS) results in an integrated line-of-sight (LOS) measurement and can be used to measure various flow properties, such as temperature and species concentration. However, the integrated nature of a TDLAS measurement can be limiting in value as a measurement technique if large gradients in flow properties exist in the flow of interest.

The technique of Tunable Diode Laser Absorption Tomography (TDLAT) has been developed at the University of Virginia to spatially resolve temperature and species concentration in high speed, high enthalpy flows. Unlike TDLAS, the TDLAT technique results in a 2D spatially resolved measurement plane. Over 2500 individual TDLAS LOS measurements are collected at numerous locations surrounding the flow of interest and then mathematically reconstructed using MATLAB's ifanbeam tomographic inversion algorithm.

When applied to a supersonic combustor model with hydrogen injection, TDLAT can be utilized to gain understanding of the combustion efficiency. The spatially resolved water vapor concentration results from the TDLAT technique can be combined with Stereoscopic PIV (which results in 3D velocity vectors) and thus the water vapor flux can be calculated. This measured flux, when compared to the known injected hydrogen flux, can be used as a direct measurement of combustion efficiency in hydrogen-air combustors. This approach is described by the following equation:

$$
\eta_{c}=\frac{\int A_{\text {ext }} n_{H_{2} O}(y, z) V_{x}(y, z) d A_{\text {exit }}}{\dot{N}_{\text {Hydrogeninjected }}}
$$

where $n_{H 2 O}(y, z)$ is the spatially resolved water number density distribution from the TDLAT measurement, $V_{x}(y, z)$ is the axial velocity distribution from the SPIV measurement, and $\dot{N}_{\text {Hydrogen, injected }}$ is the total hydrogen flux injected into the combustor. 
Validation of the TDLAT technique has been performed on the University of Virginia's flat flame burner as well as on the $\mathrm{UVaSCF}^{3,4}$. An in situ calibration of the TDLAT technique was performed by making measurements on the UVaSCF without combustion and with a known mole fraction of the water vapor (12\%) injected into the free stream. Figure 1 shows the reconstructed temperature and water vapor mole fraction at the exit plane of the combustor for a combustor total temperature of $1200 \mathrm{~K}$. A warm core with temperatures near $700 \mathrm{~K}$ is surrounded by a cooler transition layer around 550-600 K and reaching wall temperatures of approximately $500 \mathrm{~K}$. The outer temperatures near $300 \mathrm{~K}$ are indicative of the surrounding ambient room-temperature air. This core temperature around $700 \mathrm{~K}$ was found to be approximately $150 \mathrm{~K}$ lower than that found by LOS TDLAS ${ }^{3}$. Work to resolve the discrepancy is still ongoing. The water vapor mole fraction reconstruction shows a core of air with $11-12 \%$ water vapor mole fraction, in agreement with the $12 \%$ steam injected.
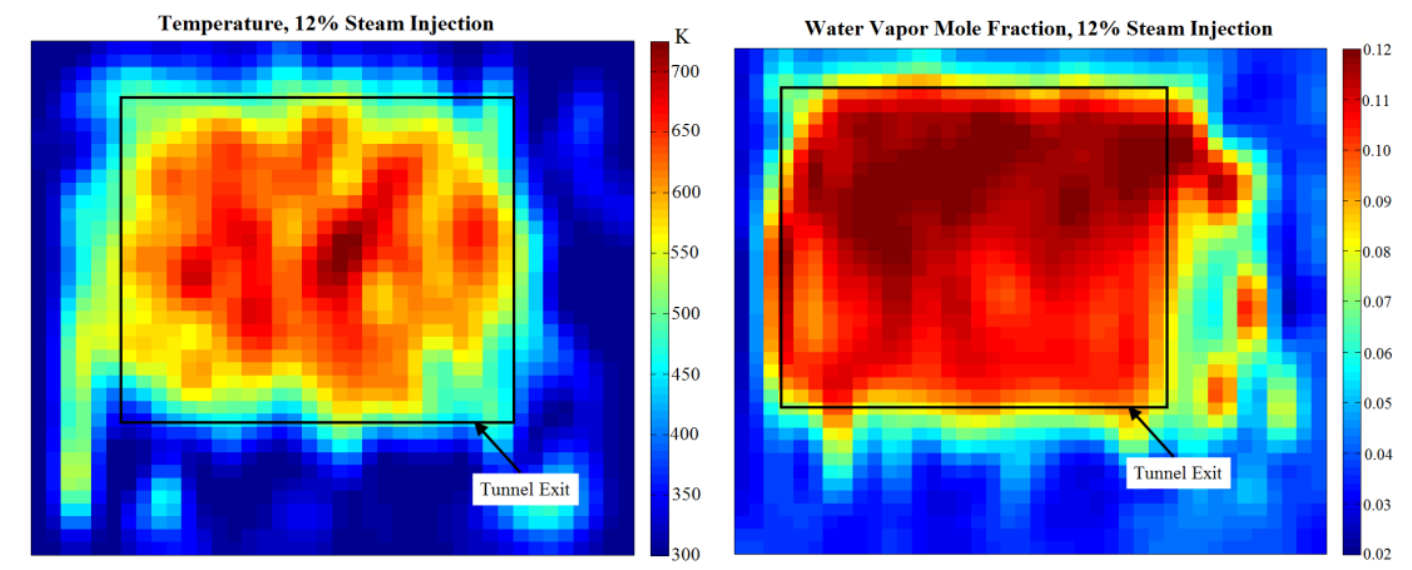

Figure 1. Temperature and mole fraction distributions from reconstruction of TDLAT measurements on UVaSCF non-combusting with $T_{0}=1200 \mathrm{~K}$ and $12 \%$ steam injection. $^{3}$

Successful implementation of the TDLAT technique in the UVaSCF has prompted an extension of the technique to more challenging experiments, with regard to experimental parameters. Of particular interest to scramjet ground testing is the ability to "scale" up in size, specifically in the magnitude of air mass flow rate. As reported herein, TDLAT has been reconfigured to make spatially resolved temperature and species concentration measurements in the NASA Langley Direct-Connect Supersonic Combustion Test Facility (DCSCTF). The DCSCTF provides ten times the flow rate of the UVaSCF, and as such the complexity of this facility provided significant challenges compared to the UVaSCF. There were three inherent challenges with applying the TDLAT technique to the DCSCTF that were encountered and overcome:

1. Larger test section area. The area of the DCSCTF exit plane is 5.5" $\mathrm{x} 5.2$ " compared to the UVaSCF's 1.0" $\mathrm{x} 1.5$ " exit plane.

2. Decreased test duration. Typical DCSCTF test times range between 30 and 40 seconds compared to hours of uninterrupted testing in the UVaSCF.

3. Harsh and noisy conditions at the DCSCTF exit plane.

The first two problems listed were anticipated and considered in the preliminary phases of this research. The third problem was encountered during active testing and was resolved in the midst of testing.

\section{Theory}

TDLAT consists of two major components: absorption spectroscopy and computed tomography. Absorption spectroscopy is used to obtain spectral information at various positions around the measurement space, and then computed tomography is used to render a $2 \mathrm{D}$ distribution of the gas properties.

\section{A. Absorption Spectroscopy}

Absorption spectroscopy is governed by the Beer-Lambert law, which states that the ratio of the transmitted intensity, $I$, to the incident intensity, $I_{0}$, is proportional to the exponential of the path length $L[\mathrm{~cm}]$ of the absorbing species (water vapor in this research) multiplied by $\kappa_{v}$, the spectral absorption coefficient [cm-1]: 


$$
I / I_{0}=\exp \left(-\kappa_{v} L\right)
$$

The spectral absorption coefficient is given by:

$$
\kappa_{v}=S(T) \cdot \varphi_{v} \cdot N_{T}
$$

where $S(T)$ is the transition line strength $\left[\mathrm{cm}^{-1} /\right.$ molecule- $\left.\mathrm{cm}^{-2}\right], N_{T}$ is the number density of the absorbing species [molecules $\cdot \mathrm{cm}^{-3}$ ], and $\varphi_{v}$ is the lineshape function [cm], approximated by a Voigt function. The Voigt function is the spectral line shape resulting from the convolution of independent Lorentzian and Doppler line broadening mechanisms:

$$
\varphi_{v}=\frac{1}{\alpha_{D}} \sqrt{\frac{\ln 2}{\pi}} K\left(\frac{\left(v-v_{0}\right)}{\alpha_{D}} \sqrt{\ln 2}, \frac{\alpha_{L}}{\alpha_{D}} \sqrt{\ln 2}\right)
$$

where $\alpha_{D}$ is the Doppler width, $\alpha_{L}$ is the Lorentz width, $v_{0}$ is the transition line center wavenumber, and $K$ is the Voigt function. The temperature dependent line strength can be calculated using:

$$
S(T)=S\left(T_{0}\right) \frac{Q\left(T_{0}\right)}{Q(T)} \exp \left[-\frac{h c E^{\prime \prime}}{k_{B}}\left(\frac{1}{T}-\frac{1}{T_{0}}\right)\right] \times\left[1-\exp \left(-\frac{h c v_{0}}{k_{B} T}\right)\right] \times\left[1-\exp \left(-\frac{h c v_{0}}{k_{B} T_{0}}\right)\right]^{-1}
$$

where $h$ is Planck's constant, $c$ is the speed of light, $k_{B}$ is Boltzmann's constant, $E$ " is the lower state energy level $\left[\mathrm{cm}^{-1}\right], v_{0}$ is the line center wavenumber of the selected transition, $T_{0}$ is the reference temperature $[\mathrm{K}]$, and $Q(T)$ is the partition function for the absorbing species. By substituting Eq. (3) into Eq. (2), rearranging, and integrating over $d v$ from $-\infty$ to $+\infty$, the integrated area under a line is given by:

$$
A=S(T) \cdot N_{T} \cdot L
$$

where $A$ is the wavenumber-integrated area $\left[\mathrm{cm}^{-1}\right]$. When Eq. (5) is substituted into Eq. (6) the following equation results:

$$
\ln \left(\frac{A}{S\left(T_{0}\right)} e^{-h c E^{\prime \prime} / k_{B} T_{0}}\left(1-e^{-h c v_{0} / k_{B} T_{0}}\right)\right)=\frac{-h c E^{\prime \prime}}{k_{B} T}+\ln \left(\frac{Q\left(T_{0}\right)}{Q(T)} N_{T} L\right)+\ln \left(1-e^{-h c v_{0} / k_{B} T}\right)
$$

For the wavenumbers and temperatures considered in this research, the $\exp \left(-h c v_{0} / K_{B} T\right)$ terms can be neglected and thus Eq. (7) is simplified to:

$$
\ln \left(\frac{A}{S\left(T_{0}\right)} e^{-h c E^{\prime \prime} / k_{B} T_{0}}\right)=\frac{-h c E^{\prime \prime}}{k_{B} T}+\ln \left(\frac{Q\left(T_{0}\right)}{Q(T)} N_{T} L\right)
$$

A so-called Boltzmann plot can be constructed using Eq. (8) and several transitions. For each given transition, the absorption spectra are analyzed and the integrated areas under the transitions are calculated. The left-hand-side of Eq. (8) and the transition's known lower state energy form an ordered pair. A linear fit is then performed on the collection of ordered pairs and the temperature and number density of the absorbing transition can be determined from the fit parameters.

\section{B. Tomographic Image Reconstruction}

Computed tomography is the process of capturing the internal structure of an object by mathematically reconstructing a series of measurements collected through the object. This process is most familiarly associated with the medical field's Computer Axial Tomography (CAT) scan. In this case, a series of LOS X-ray images are collected from multiple angles and positions around the object of study. The LOS X-ray measurements are then computationally reconstructed to form a 2D cross-sectional image of the object. Analogously, multiple 1D TDLAS 
LOS measurements are collected from multiple angles and positions around the object of study and are computationally reconstructed to form a $2 \mathrm{D}$ image of the object.

If the measurements are made from a number of evenly spaced and symmetric locations around the periphery of the object of interest, a transform-based reconstruction method can be used. ${ }^{5}$ In this research, the filtered backprojection algorithm is used, which is based on the Fourier slice (or central slice) theorem.

To collect the TDLAS measurements, an infrared diode laser revolves around the object of interest. At discrete and evenly spaced locations along its circular path, the laser source stops for a measurement, such as locations $s 1$ and $s 2$ in Figure 2. At each of these fan locations, the laser source then rotates through a discrete set of angles creating a fanbeam, as illustrated in Figure 2. Along each ray a single LOS TDLAS measurement is made.

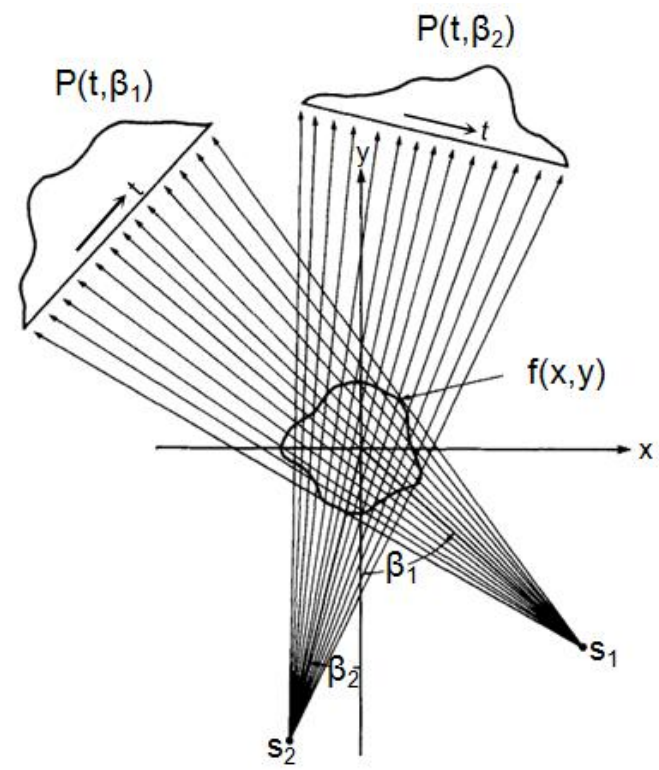

Figure 2. Schematic of multiple fan beams being collected around the periphery of an object. ${ }^{6}$

After the TDLAS measurements have all been collected, each transition's integrated absorbance is determined for each LOS spectrum. A matrix can then be formed, organizing the integrated areas, denoted as $\boldsymbol{A}_{i, j, k}$, according to three indices: the ray number, fan number, and transition (or wavelength) index. The $\boldsymbol{A}$ matrix is the variable to be reconstructed. For $\mathrm{k}=1$ to the number of transitions, a filtered back-projection algorithm is used to obtain a $2 \mathrm{D}$ reconstruction of each $\mathrm{A}(:, ;, \mathrm{k})$. These $2 \mathrm{D}$ matrices correspond to the actual physical measurement plane, mapping out the area under the transition as a function of spatial location. At each point in space, i.e. each pixel, the LHS's of Eq.(8) are plotted against their corresponding lower energy states, and this Boltzmann plot is utilized to calculate the temperature and water vapor concentration.

\section{Experimental Setup}

The DCSCTF is capable of testing ramjet and scramjet combustor models at conditions simulating flight from Mach 4 to Mach 8. A Mach 5 flight-enthalpy has been simulated for this research. Hydrogen combustion with oxygen-enriched air is used to heat the test gas to achieve the required conditions. The facility can operate with a variety of nozzles and (sc)ramjet models. The DCSCTF is ideal for research in mixing, ignition, flameholding, and combustion characteristics ${ }^{7}$. The DCSCTF is shown in Figure 3. 


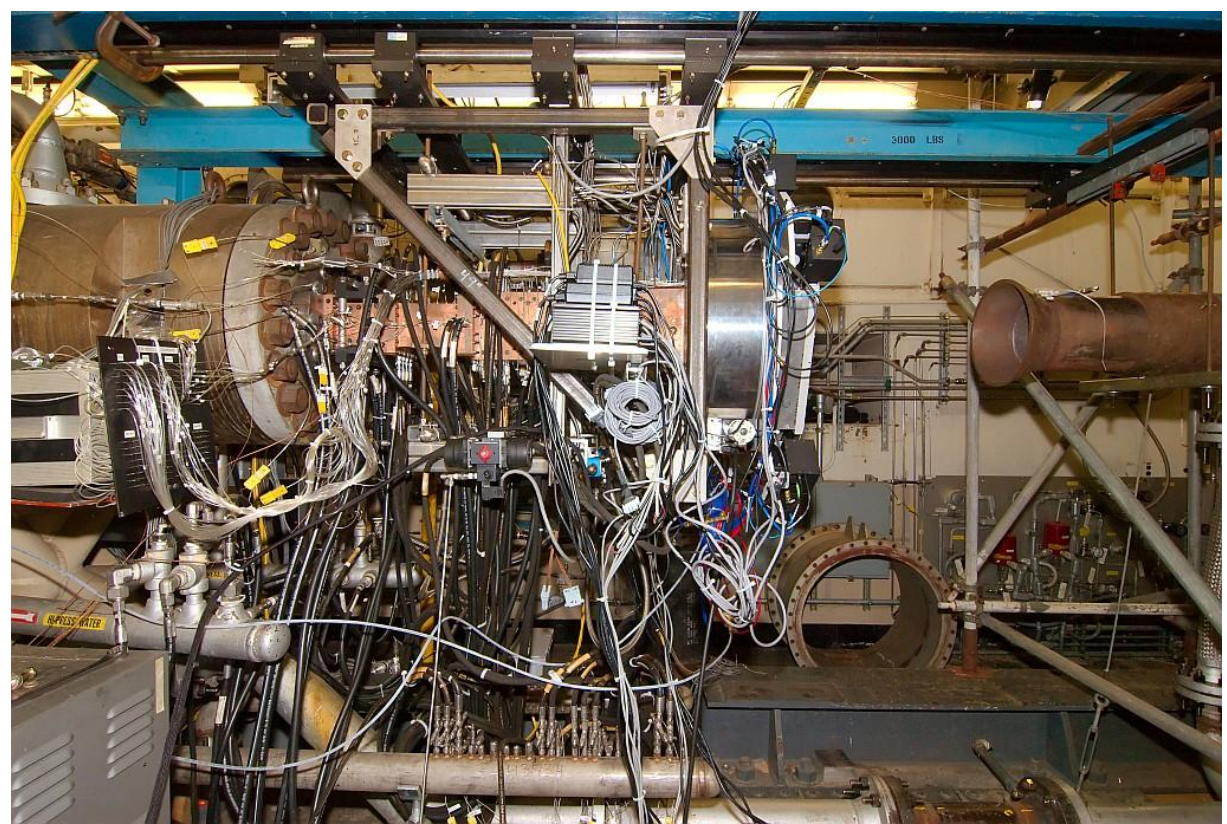

Figure 3. NASA Langley Direct-Connect Supersonic Combustion Test Facility with UVA TDLAT system installed.

The TDLAT hardware is mounted to the DCSCTF inside the test cell, seen in Figure 3. During operation and between tests, the test cell remains closed. Therefore, although the TDLAT hardware is located inside the test cell, the TDLAT computer (runs data acquisition and motion control software) and the diode lasers are located in an adjacent room. The power cables, data cables, and optical fibers are fed into the test cell through a small hole in the wall as seen in Figure 4. This allows the user to actively control the TDLAT equipment remotely.

DIRECT-CONNECT SUPERSONIC COMBUSTION TEST FACILITY

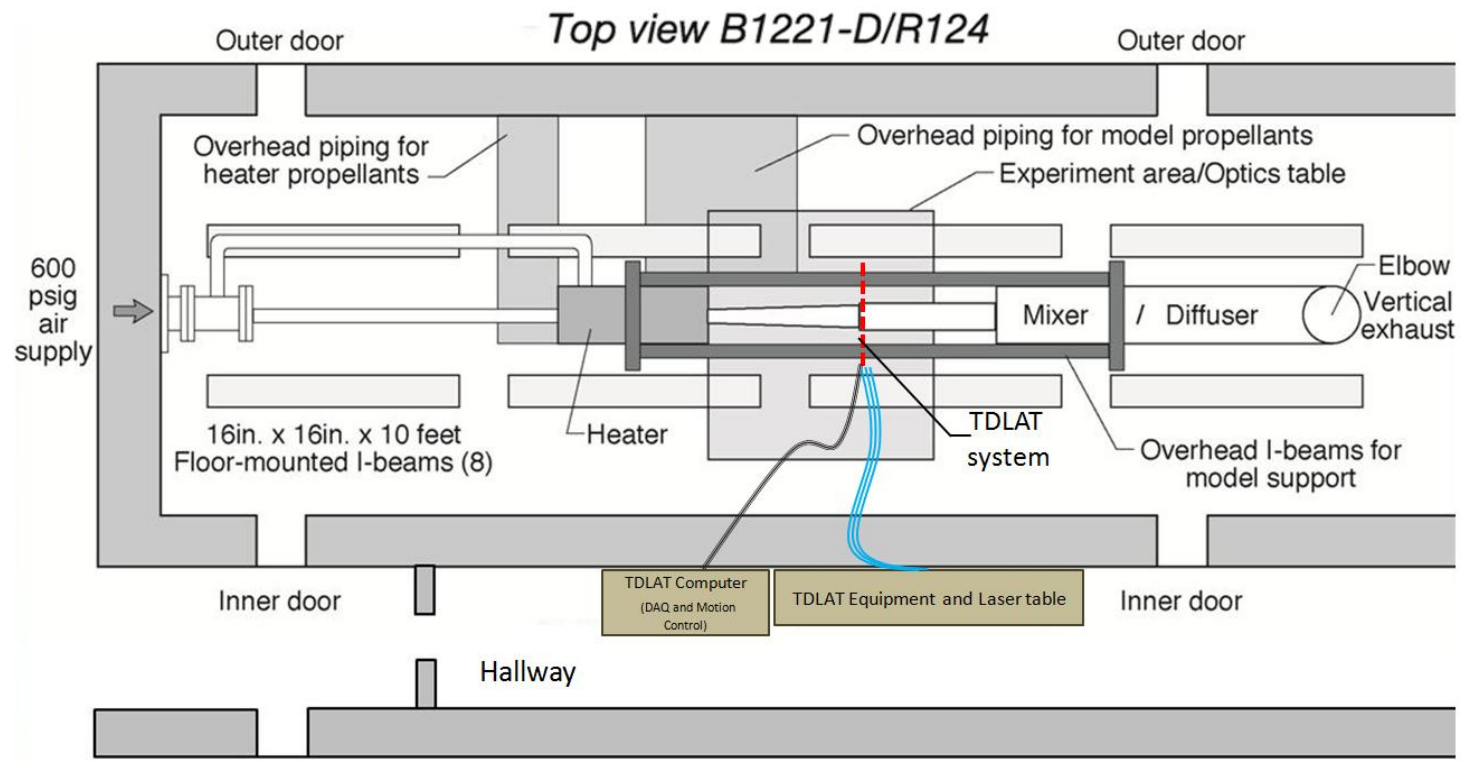

Figure 4. Schematic of Direct-Connect Supersonic Combustion Test Facility and location of TDLAT Equipment. 
The TDLAT technique makes use of three NTT Electronics polarization maintaining (PM) fiber coupled distributed feedback (DFB) lasers (NLK1E5GAAA and NLK1B5EAAA). The selection of the line transitions to use in this research was guided by X. Zhou et al. ${ }^{8}$ and consisted of four main criteria:

1) Sufficient absorption required for high signal-to-noise ratio (SNR) measurements.

2) Transitions should be selected to minimize interference from ambient (low-temperature) $\mathrm{H}_{2} \mathrm{O}$, i.e. E" $\geq$ $1700 \mathrm{~cm}^{-1}$ for the selected transitions.

3) The transitions must have sufficiently different lower state energies, $E^{\prime \prime}$, to yield a peak height ratio that is sensitive to temperature (in the range 1000-2500K), i.e. $\left|\mathrm{E}_{1}{ }^{\prime \prime}-\mathrm{E}_{2}{ }^{\prime \prime}\right| \geq 700 \mathrm{~cm}^{-1}$.

4) The selected transitions must be isolated from nearby transitions.

Table 1. Transitions used in TDLAT system and their spectroscopic parameters. ${ }^{9,10}$

\begin{tabular}{|c|c|c|c|c|}
\hline Transition & $\begin{array}{c}\text { Linecenter Wavenumber } \\
v_{0}\left(\mathrm{~cm}^{-1}\right)\end{array}$ & $\begin{array}{c}\text { Transition Reference } \\
\text { Intensity } \\
\mathrm{S}_{\mathrm{ref}}\left(\mathrm{cm}^{-1} / \mathrm{molecule} \cdot \mathrm{cm}^{-2}\right)\end{array}$ & $\begin{array}{c}\text { Lower State Energy } \\
\text { Level } \\
\mathrm{E}^{\prime \prime}\left(\mathrm{cm}^{-1}\right)\end{array}$ & $\begin{array}{c}\left|\mathrm{E}_{\mathrm{i}}{ }^{\prime \prime}-\mathrm{E}_{\mathrm{i}+1^{\prime \prime}}\right| \\
\left(\mathrm{cm}^{-1}\right)\end{array}$ \\
\hline $\mathrm{KB} 1$ & 7185.60 & $7.95 \mathrm{E}-22$ & 1045.06 & -- \\
\hline $\mathrm{KB} 2 *$ & $7444.35 / 7444.37$ & $4.06 \mathrm{E}-23$ & 1787.00 & 741.94 \\
\hline $\mathrm{KB} 3 \mathrm{a}$ & 7471.617 & $7.96 \mathrm{E}-26$ & 2952.39 & 1165.39 \\
\hline $\mathrm{KB} 3 \mathrm{~b}$ & 7472.22 & $7.85 \mathrm{E}-26$ & 2981.36 & 1194.36 \\
\hline
\end{tabular}

*This transition consists of two transitions spaced $.02 \mathrm{~cm}-1$ apart. They are essentially indistinguishable at the test conditions reported here, so weighted $\mathrm{S}_{\mathrm{ref}}$ and $\mathrm{E}$ " are given.

The transitions selected for use this experiment are given in Table 1. Note that the designations of the absorption transitions used in this paper are internal and used only for convenience in referencing the transitions.

The four transitions identified in Table 1 were found to have sufficiently favorable characteristics for this research based on the aforementioned criteria. However, they were inaccessible within a single laser scan. For this reason, three lasers were used. KB1 \& KB2 are best suited for a lower temperature range, and KB2 \& KB3a,b are best suited for a higher temperature range. Data are collected with all 3 lasers, in a time-multiplexed mode, for the full duration of the experiment. Depending on the data quality and SNR observed during post-processing, two, three, or four of the transitions are utilized in the Boltzmann plot. The lasers were temporally multiplexed using a MEMS-type fiber optical switch system. While the KB1 laser is switched on and scans over the first transition, the KB2 and KB3 lasers are switched off. After KB1 has scanned over its prescribed range, it switches off and KB2 is switched on. Lastly, KB2 is switched off and KB3 is switched on. This process is repeated each time a measurement is collected. In order to make the measurements as close to simultaneous as possible, each trio of laser scans was accomplished in $10 \mathrm{~ms}$.

The optics are housed in a Tomographic Emitter-Detector (TED) box and a schematic is shown in Figure 5. The near infrared light is brought into the box through the PM fiber and is immediately passed through a collimating lens. The beam is collimated to a $1 \mathrm{~mm}$ diameter. It then passes through an aperture slightly larger than $1 \mathrm{~mm}$. The collimated beam travels past a small right angle prism mirror, and finally out another aperture before exiting the TED box. It then travels through the test gas and hits an arc covered in a retroreflective material. The beam is then retroreflected, i.e. the beam is reflected back onto its original path, only now the beam begins to diverge conically. The spreading beam passes again through the test

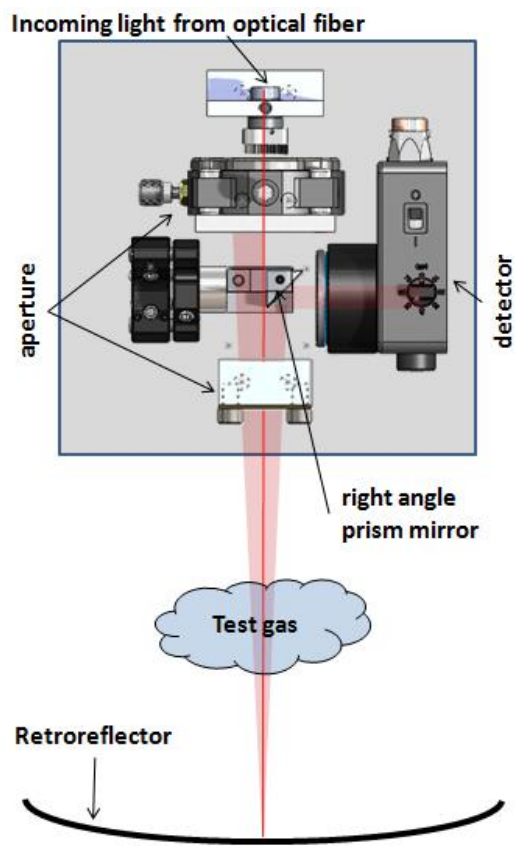

Figure 5. Schematic of the Tomographic Emitter-Detector (TED) box.

gas and back into the TED box through an aperture. The beam's diameter is larger than previously, and the spread beam impinges on the right angle prism mirror. This portion of the beam is then directed through a bandpass filter (to filter out flame luminosity), a lens, and finally into an InGaAs detector. To create the fan beam as mentioned previously, a TED box is mounted to a Velmex rotational stage, allowing it to rotate through an entire fan. The TED 
box is coated with Aeroglaze Z306, an absorptive polyurethane paint, to minimize unintentionally scattered IR light. Each TED box, as well as the volume contained by the retroreflectors, adapter plate, and top lid, is purged with dry air to minimize ambient water vapor absorption.

As discussed previously, there were three major challenges faced when applying the TDLAT technique to the DCSCTF. First, the larger test section area called for hardware modifications. The original TDLAT experimental hardware used in the UVaSCF consisted of a single TED box mounted to a smaller Newport rotational ring which rotated incrementally $360^{\circ}$ around the tunnel exit. The Newport rotational ring used for the tests conducted on the UVaSCF measured approximately 7" internal diameter, while the customized Newport rotational ring used for the DCSCTF measured approximately 19" internal diameter. Also noteworthy is that the UVaSCF has a vertical test section which allowed the TDLAT rig to be mounted horizontally. The DCSCTF has a horizontal test section, requiring the TDLAT rig to be mounted vertically. Figure 6 shows a comparison between the TDLAT system on the UVaSCF and the TDLAT system on the DCSCTF.

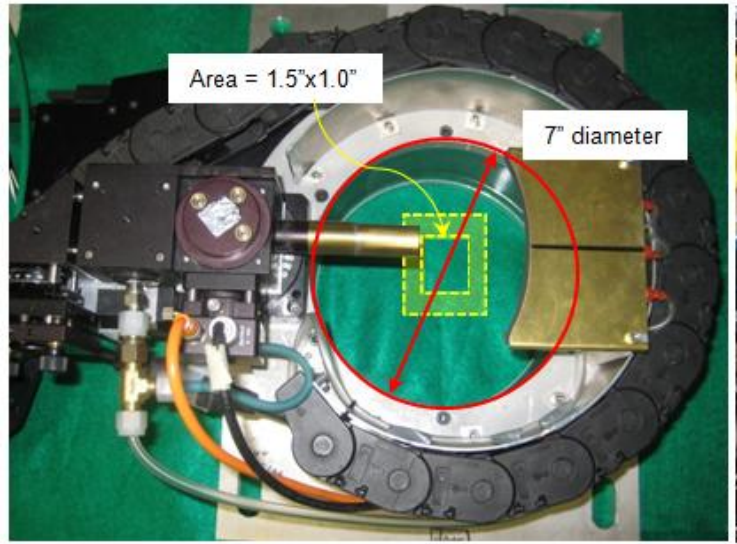

(a)

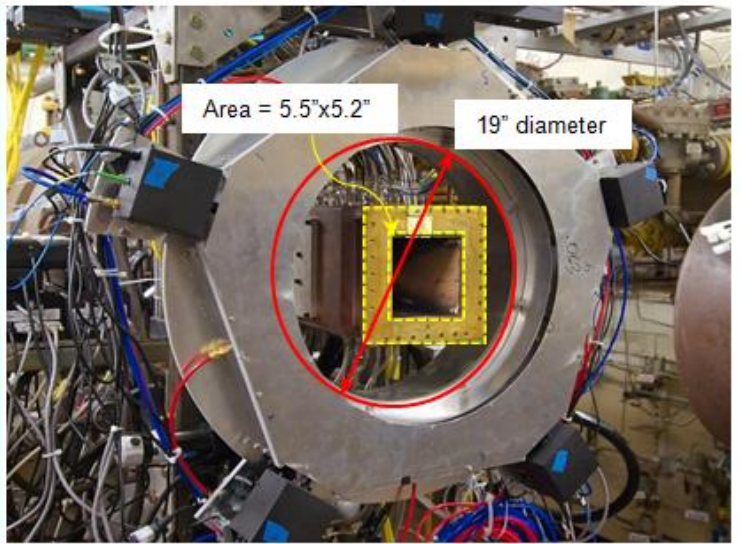

(b)

Figure 6. (a) TDLAT system used for measurements on UVaSCF and (b) TDLAT system for measurements on DCSCTF.

The second challenge, the short test duration of the DCSCTF, called for both software and hardware modifications. The 50 second maximum test time is limited by the temperature of the un-cooled copper hardware on the DCSCTF rig. This required streamlining of the motion control and data acquisition software used in the UVaSCF. Various portions of the LabVIEW 8.6 code were condensed or removed all together if unnecessary. Much of the extraneous code was for data communication between the PC and the motion controllers and was able to be replaced with simple TTL signals. The shorter test duration also motivated the decision to increase the number of TED boxes from one to five. This reduces the required test duration by the same factor. Figure 7 shows the modified TDLAT system with the top lid removed.
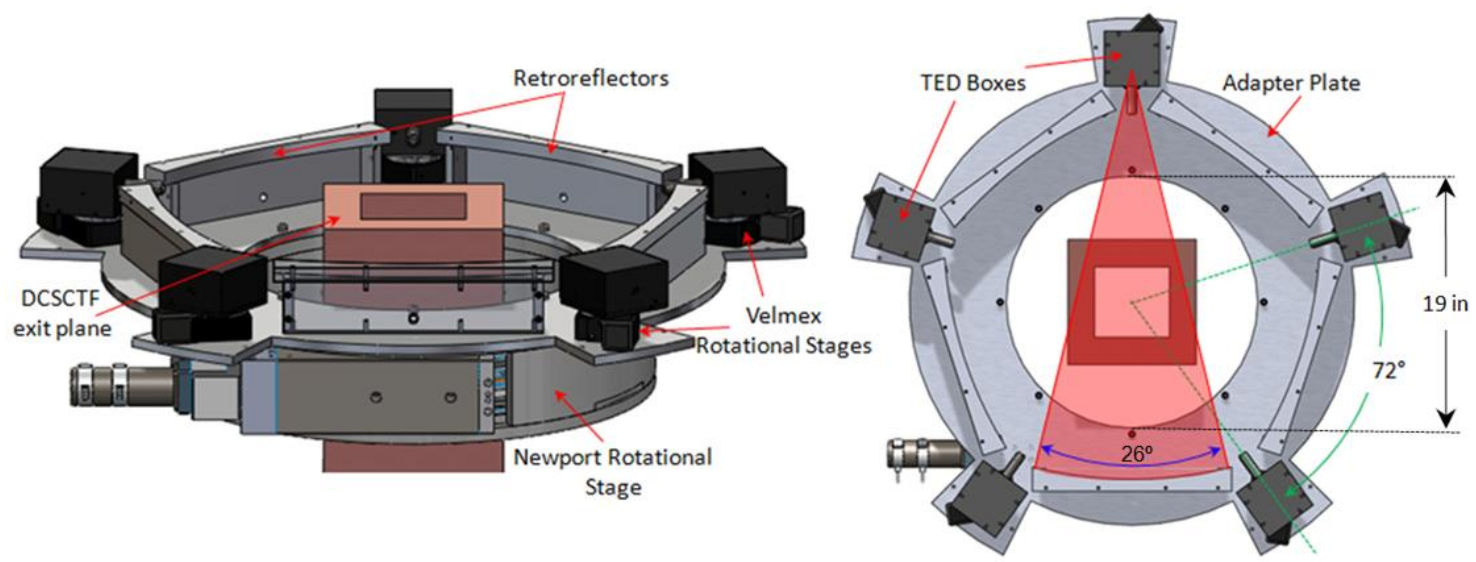

Figure 7. SolidWorks model of the TDLAT system. 
As seen in Fig. 7, an adapter plate is used to interface the Newport RTM660 rotational ring with the TED boxes and retroreflector hardware. This allows the assembly to rotate $360^{\circ}$ around the DCSCTF. However, due to the angular symmetry of the five TED boxes, the plate needs only to rotate $72^{\circ}$ for complete imaging. Attached to the adapter plate are five Velmex B5990TS rotational stages and five TED boxes. The Velmex rotational stages allow each TED box to rotate and create a fanbeam. As seen in Fig. 7, $26^{\circ}$ of rotation was needed to ensure the fanbeam encompasses the DCSCTF exit plane.

Motion and laser control have been optimized to allow for data collection during the short test duration. Each fan is composed of 21 rays which are incremented by $1.3^{\circ}$, consequently scanning a total of $26^{\circ}$. Each fan is separated by $9^{\circ}$, requiring a total of 8 data sets (each with 5 fans) to complete the database. A complete uninterrupted database requires between 6 and 7 minutes of test time, and thus the testing must be segmented. One data set, consisting of 5 fans of data, was collected during each tunnel operation. The start point of the fans during next test is then offset $9^{\circ}$ from the previous tunnel run. This requires 8 tunnel runs for a complete database, which is well within the test capabilities of the DCSCTF on a single day.

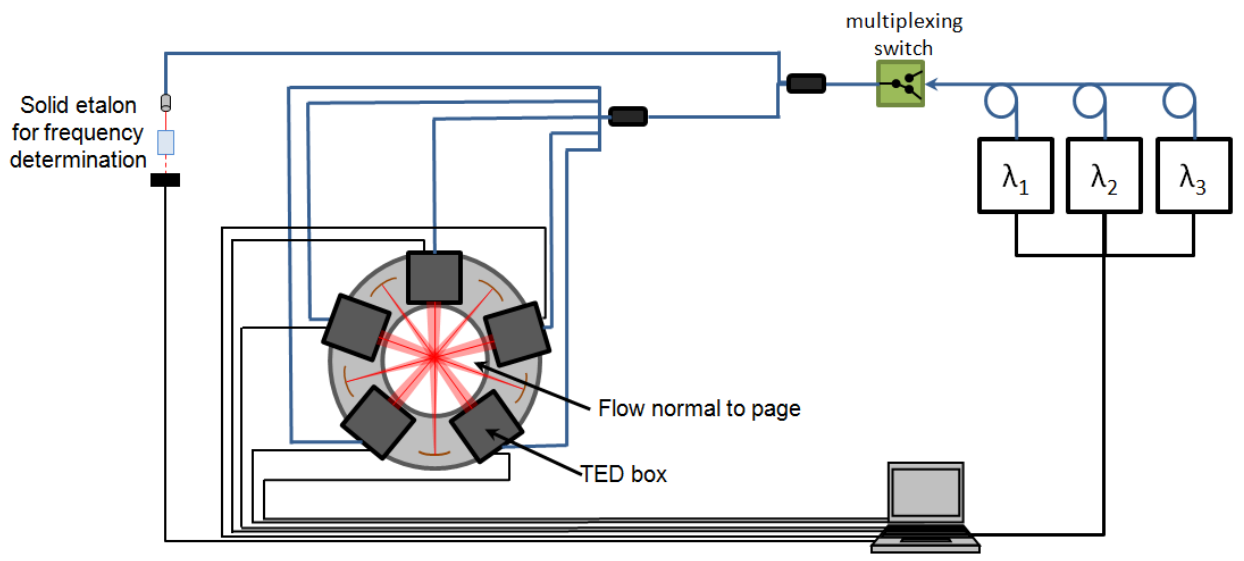

\section{Figure 8. Schematic of TDLAT system.}

The noisy environment of the DCSCTF created the need for several LOS measurements to be collected at each location and averaged. Figure 9 shows sample data from a measurement near the center of the test plane. The single LOS measurement shows that the peak-to-peak amplitude of the noise is on the same order as the signal itself. Although the noise looks to be quasi-periodic, its periods do not appear to be coupled to the period of the sample. As the signals are averaged over a greater number of samples, a clear absorption feature is revealed.

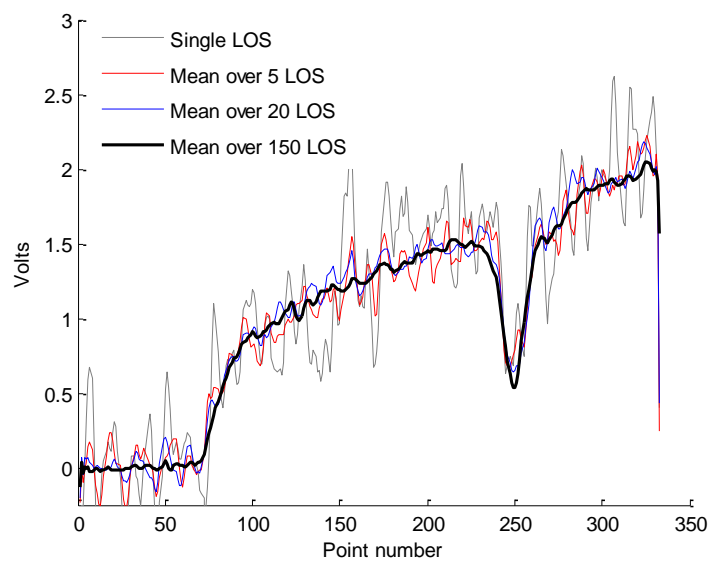

Figure 9. Single and averaged LOS sample data.

For this research, 150 individual LOS measurements are collected and averaged for each laser and at each location. The data is acquired at 100,000 samples/sec, with 150,000 samples/channel. Therefore 1.5 seconds is spent collecting the data at each location. 


\section{Results}

Prior to the installation of this system on the DCSCTF, the TDLAT system was tested on a NASA Langley flat flame Hencken burner, shown in Figure 10. Multiple tests were performed, including the measurement of an opaque object. This object was positioned on the burner surface and in the laser plane as shown in Fig. 10 (the flame was not utilized here).

A full set of data was collected based on the methods previously described. When the fanbeam path crosses the object, no signal is returned. This is slightly different than how laser absorption spectroscopy operates, as with spectroscopy the amount of absorption is dependent on the quantity and temperature of the water vapor.

This opaque object test is essentially a binary version of absorption spectroscopy, with only 1's (signal is returned) and 0's (signal is blocked by object). A 0 or 1 is recorded for each ray in each fan and collected into a matrix called a sinogram. The reconstruction is then performed on this sinogram using the Matlab function "ifanbeam.m". Figure 11 shows the sinogram and the tomographic reconstruction of the opaque object. We see the object is the same shape and oriented in the same direction as in Figure 10. The rippling seen in this reconstruction, as well as the fact that the object appears a non-uniform color are data artifacts and are directly related to the use of a binary signal. The actual use of TDLAT is for high enthalpy flowfields, not solid objects, and thus these data artifacts will not necessarily be encountered.

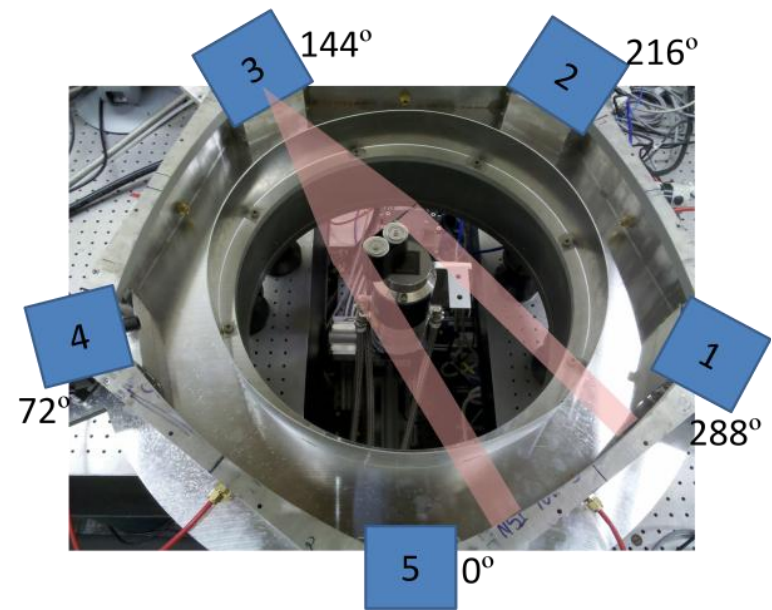

Figure 10. TDLAT system mounted to NASA Langley flat flame Hencken burner. Opaque object positioned on burner surface.

In addition to this solid object test, data has been taken on the Hencken burner with the flame operational. Three different equivalence ratios, $\Phi=0.3,1.2,2.0$ were measured. Figure 12 shows sample spectra from the $\Phi=1.2$ condition. The measured spectra along with their Voigt fits are shown for all four transitions. These spectra are from a ray in the center of a fan beam, so much of the ray's pathlength is within a relatively high temperature range. The favorable quality of the collected spectra was promising and future research will focus on completing the tomographic reconstructions. The resulting TDLAT temperature and water vapor concentration distributions will be compared to previous temperature measurements via CARS by another research group.
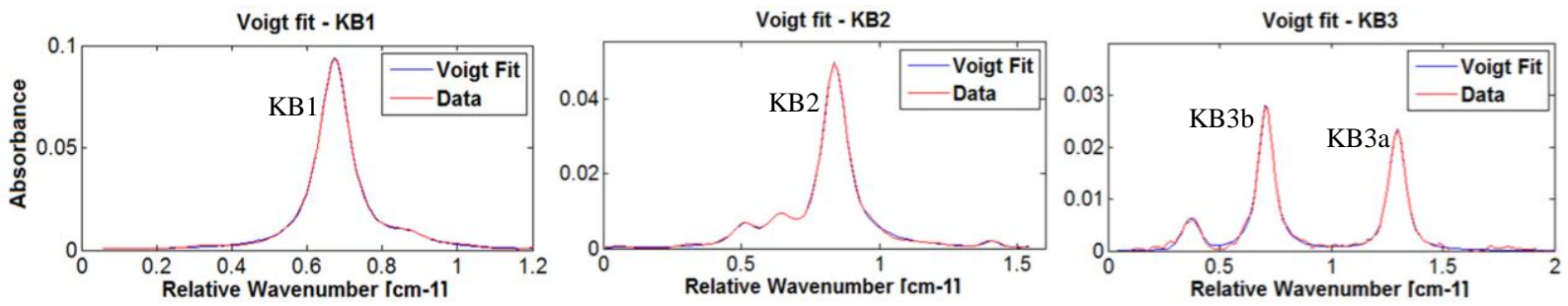

Figure 12. Sample spectra from a center ray of TDLAT measurement on Hencken burner with $\Phi=1.2$. 
The TDLAT test rig was then mounted to the DCSCTF. Heated, non-combusting measurements, as well as combustion measurements at an equivalence ratio of 0.5 , have been collected at a facility condition simulating flight at Mach 5. Final tomographic reconstruction of the data is not yet complete, but preliminary analysis has been conducted. Table 2 summarizes the Mach 5 test conditions.

Table 2. Test Conditions during TDLAT measurements on DCSCTF.

\begin{tabular}{|c|c|c|c|c|c|c|c|}
\hline $\begin{array}{c}\text { Run } \\
\text { No. }\end{array}$ & Date & $\begin{array}{c}\text { Avg. Period } \\
{[\text { seconds] }}\end{array}$ & $\begin{array}{c}\mathbf{P}_{\mathbf{0}} \\
{[\mathbf{p s i a}]}\end{array}$ & $\begin{array}{c}\mathbf{T}_{\mathbf{0}} \\
{[\mathbf{R}]}\end{array}$ & $\begin{array}{c}\mathbf{H}_{\mathbf{T} 0} \\
{\left[\mathbf{B T U} / \mathbf{l b}_{\mathbf{m}}\right]}\end{array}$ & $\begin{array}{c}\mathbf{O}_{\mathbf{2}} \\
{[\mathbf{\%}]}\end{array}$ & $\mathbf{\Phi}$ \\
\hline 87 & $8 / 16 / 2012$ & $38.5-46.5$ & 95.5 & 2122 & 581.1 & 20.29 & 0.587 \\
\hline 88 & $8 / 16 / 2012$ & $34.0-44.0$ & 96.2 & 2101 & 573.8 & 21.32 & 0.520 \\
\hline 89 & $8 / 16 / 2012$ & $34.0-44.0$ & 96.2 & 2113 & 578.2 & 20.82 & 0.555 \\
\hline 90 & $8 / 16 / 2012$ & $36.0-46.0$ & 96.0 & 2125 & 582.2 & 20.91 & 0.549 \\
\hline 91 & $8 / 16 / 2012$ & $39.0-49.0$ & 96.6 & 2132 & 583.9 & 20.98 & 0.562 \\
\hline 92 & $8 / 16 / 2012$ & $40.0-50.0$ & 96.6 & 2131 & 583.8 & 21.14 & 0.526 \\
\hline 93 & $8 / 16 / 2012$ & $38.5-48.5$ & 96.4 & 2132 & 584.2 & 20.86 & 0.552 \\
\hline 94 & $8 / 16 / 2012$ & $39.0-49.0$ & 96.8 & 2162 & 593.9 & 20.93 & 0.510 \\
\hline 95 & $8 / 16 / 2012$ & $40.0-50.0$ & 97.1 & 2137 & 585.9 & 20.91 & 0.533 \\
\hline 96 & $8 / 16 / 2012$ & $40.0-50.0$ & 97.1 & 2142 & 587.7 & 20.85 & 0.545 \\
\hline & & & & & & & \\
\hline 98 & $8 / 20 / 2012$ & $40.0-50.0$ & 98.8 & 2163 & 595.0 & 20.88 & 0.543 \\
\hline 99 & $8 / 20 / 2012$ & $25.0-35.0$ & 97.8 & 2154 & 592.0 & 20.88 & 0.545 \\
\hline 01 & $8 / 20 / 2012$ & $28.0-38.0$ & 97.7 & 2149 & 590.4 & 20.93 & 0.536 \\
\hline 02 & $8 / 20 / 2012$ & $35.0-45.0$ & 97.6 & 2132 & 584.3 & 21.03 & 0.547 \\
\hline
\end{tabular}

As mentioned previously, a complete data set is captured with 8 tunnel runs, resulting in a data set with 40 fans and $9^{\circ}$ between each fan. As seen in Figure 13, this creates a dense measurement within the DCSCTF exit area. Originally, a finer resolution was planned utilizing 16 tunnel runs with a fan-increment angle of $4.5^{\circ}$, but only 8 tunnels runs were completed on 8/16/12 due to facility complications. An attempt to collect the remaining 8 sets of data was made on $8 / 20 / 12$, but only 4 were successful due to the same facility complications. For the current work, a reconstruction is being done using the 8 tests from $8 / 16 / 2012$, while future work will incorporate the remaining four tests from 8/20/12. More detail regarding the facility configuration and conditions can be found in Reference 11 . A TDLAT measurement can also be reconstructed using subsets of the dense data. This includes the case of data from a single tunnel run. Future research plans include completing a coarse reconstruction from each of the 12 successful tests and evaluating their likeness.

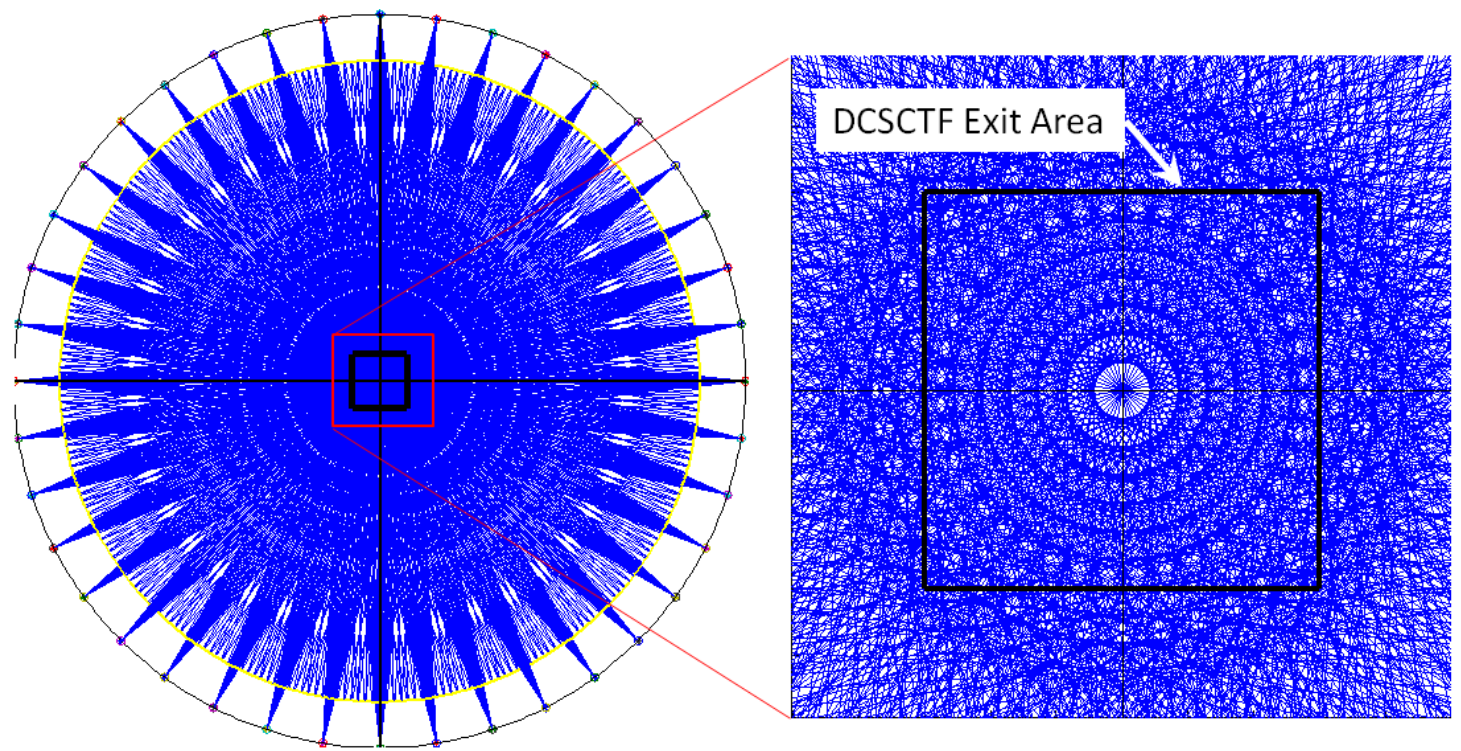

Figure 13. Schematic of measurements from 8 tunnel runs. 21 rays in each fan, 40 fans separated by 9 degrees. 
LOS temperatures have been calculated using the experimentally obtained data. Figure 14 shows the fitted spectra measured by KB1, KB2, and KB3 at a cold ray. As seen in Fig. 14, this ray passes through the edge of the tunnel exit area and presumably would see a relatively low temperature. A Boltzmann plot is shown with the calculated value of $938 \mathrm{~K}$. Likewise, LOS data is analyzed for a hot ray and shown in Figure 15. The hot ray results in a calculated temperature of $1333 \mathrm{~K}$. While these calculated temperatures are LOS values, and thus pathintegrated, they do show the expected temperature trends.
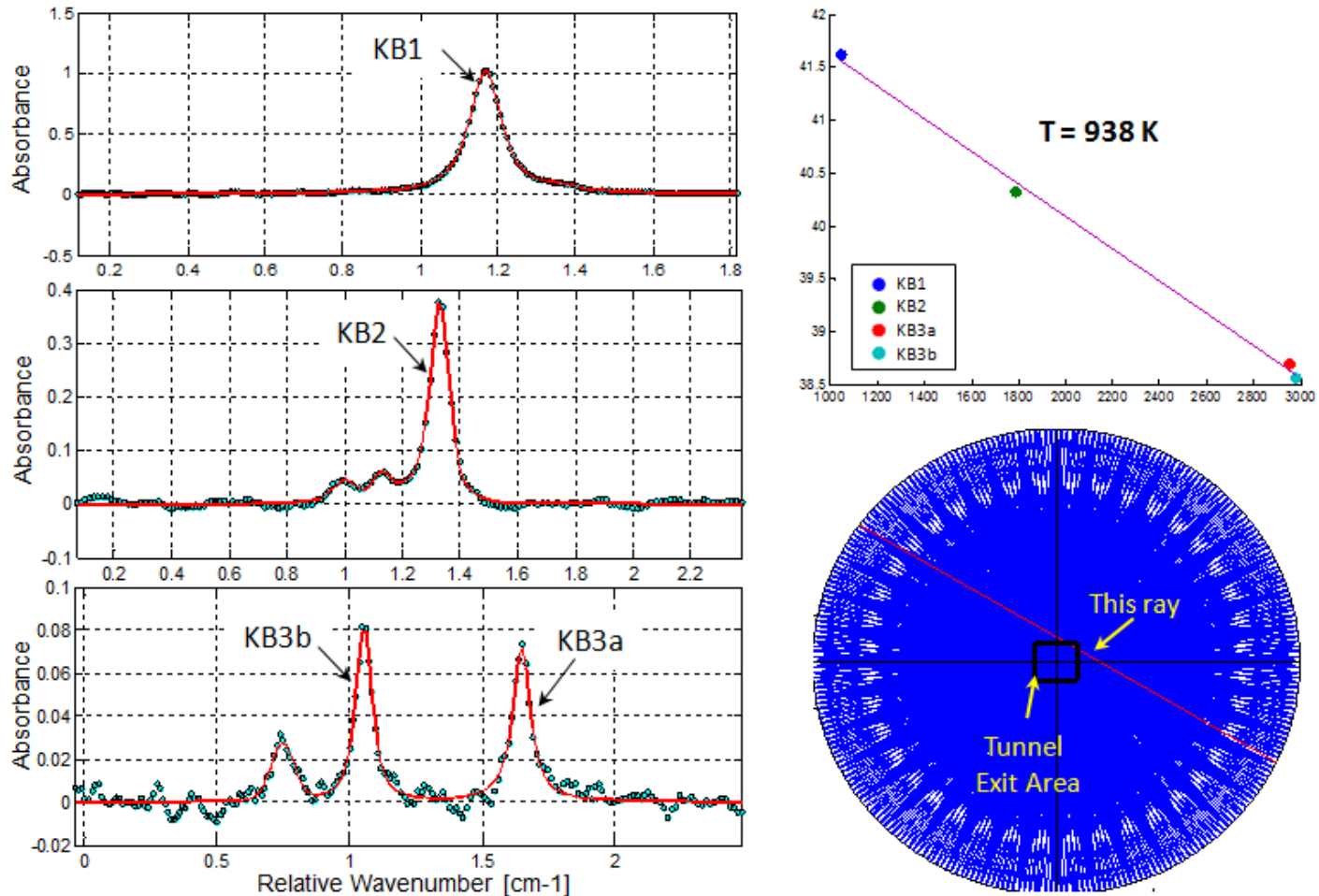

Figure 14. Fitted spectra, calculated temperature, and location of a cold ray.
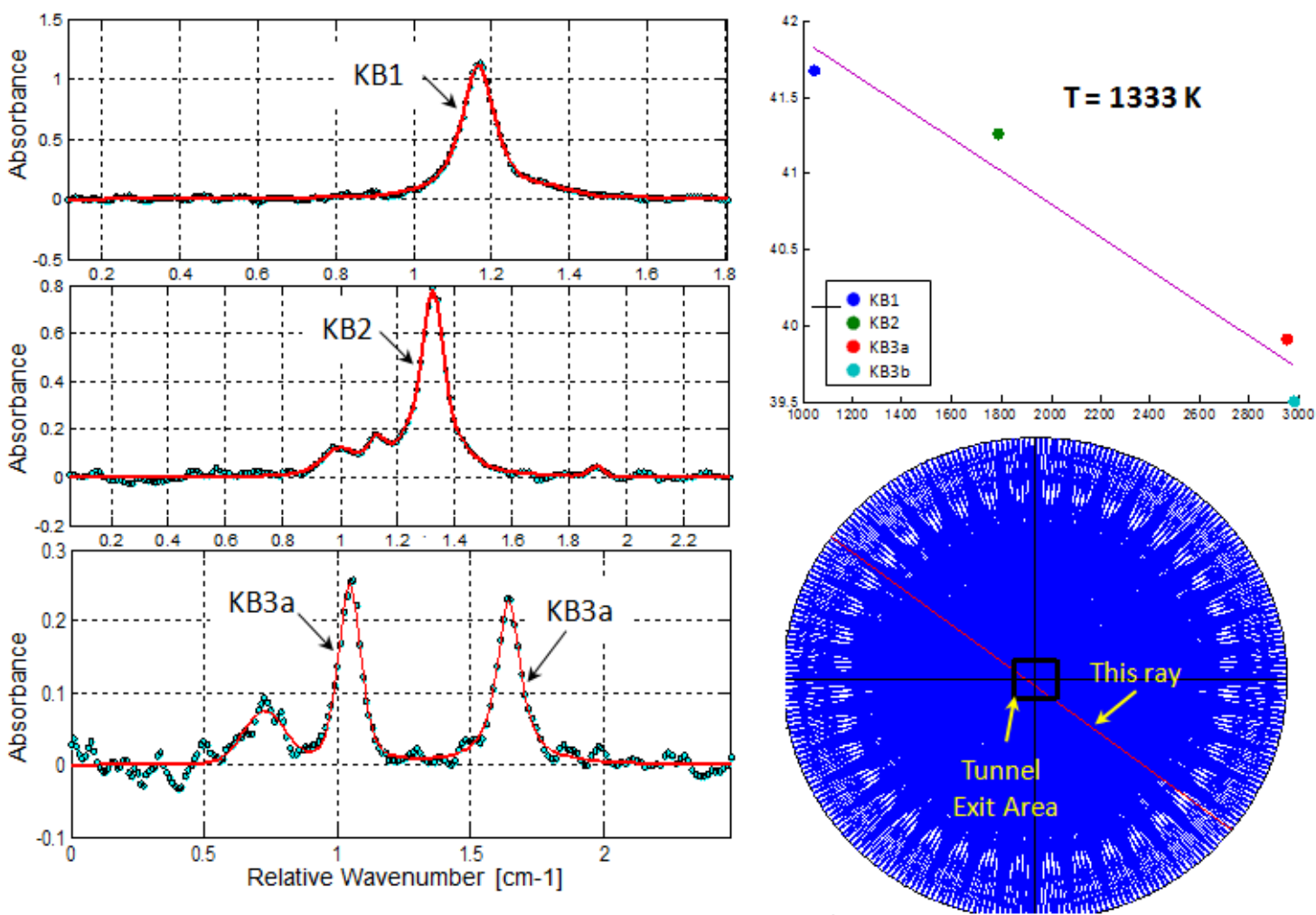

Figure 15. Fitted spectra, calculated temperature, and location of a hot ray. 


\section{Conclusion}

The TDLAT technique has been modified for application to the NASA Langley DCSCTF. Modification of software and hardware allowed for successful implementation of a larger scale testing. Twelve datasets we successfully captured and eight of these will be used for initial analysis (for symmetry). Preliminary analysis of LOS measurements show promising results and give confidence in the potential of the TDLAT technique.

Future work will first be focused on completing the tomographic reconstructions of temperature and water vapor concentration distribution. Studies will also be performed analyzing the impact of the density of the LOS data, i.e. how many LOS are utilized. More rigorous comparisons and quantitative assessment of the CFD and experimental results will be completed.

\section{Acknowledgements}

This research was supported by NASA Contract NNL11AB32P (Rick Gaffney, technical monitor) and the National Center for Hypersonic Combined Cycle Propulsion Grant FA9550-09-1-0611, supported by NASA and the AFOSR (Rick Gaffney and Chiping Li, technical monitors). Kristin Busa would like to acknowledge fellowship support from the National Science Foundation and the Virginia Space Grant Consortium. The authors would like to thank Barry Lawhorne, Michael Raiford, and Jeffrey Collins with NASA Langley Research Center for their operation of the DCSCTF, and Roger Reynolds with the University of Virginia for his support in the fabrication of the TDLAT hardware.

\section{References}

${ }^{1}$ Allen, M.G., "Diode laser absorption sensors for gas-dynamic and combustion flows," Meas. Sci. Technol., Vol. 9, 1998, pp. 545-562.

${ }^{2}$ Hanson, R. K., Kuntz, P. A., and Kruger, C. H., "High-resolution Spectroscopy of Combustion Gases using a Tunable IR Diode Laser," Applied Optics, Vol. 16, No. 8, 1977, pp. 2045-2048.

${ }^{3}$ Busa, K., Bryner, E., McDaniel, J.C., Goyne, C.P., Diskin, G., Smith, C., "Demonstration of Capability of Water Flux Measurement in a Scramjet Combustor using Tunable Diode Laser Absorption Tomography and Stereoscopic PIV," AIAA-20111294, Jan. 2011.

${ }^{4}$ Bryner, E., Busa, K., McDaniel, J.C., Goyne, C.P., Diskin, G., "Spatially Resolved Temperature and Water Vapor Concentration Distributions in a Flat Flame Burner by Tunable Diode Laser Absorption Tomography," AIAA-2011-1291, Jan. 2011.

${ }^{5}$ Bryner, E., Sharma, M.G., Goyne, C.P., McDaniel, J.C., Snyder, M.C., Krauss, R.H., and Martin, E.F., "Tunable Diode Laser Absorption Technique Development for Determination of Spatially Resolved Water Concentration and Temperature," AIAA 2010-299, Jan. 2010.

${ }^{6}$ Kak, A.C., and Slaney, Malcolm., Principles of Computerized Tomographic Imaging, Society of Industrial and Applied Mathematics, 2001.

7“"The Langley Direct-Connect Supersonic Combustion Test Facility Brochure.” Hypersonic Airbreathing Propulsion Branch. NASA Langley Research Center, 11 Oct. 2005. Web. 1 Mar. $2012 . \quad<$ http://hapbwww.larc.nasa.gov/Public/Facilities/Dcsctf/Wte_info/DCSCTF_brochure.html>.

${ }^{8}$ Zhou, X., Jefferies, J.B., and Hanson, R.K., "Development of a fast temperature sensor for combustion gases using a single tunable diode laser," Applied Physics B, Vol. 81, 2005, pp. 711-722.

${ }^{9}$ Rothman, L.S., Gordon, I.E., Barber, R.J., Dothe, H., Gamache, R.R., Goldman, A., Perevalov, V., Tashkun, S.A., and Tennyson, J., "HITEMP, the high-temperature molecular spectroscopic database," Journal of Quantitative Spectroscopy and Radiative Transfer, 111:21392150, 2010.

${ }^{10}$ Goldenstein, C., (Stanford University), personal communication.

${ }^{11}$ Capriotti, D.P., and DePiro, M.J., "Testing of the Durable Combustor Rig in the NASA Langley Direct Connect Facility," JANNAF $45^{\text {th }}$ CS $/ 33^{\text {rd }}$ APS $/ 33^{\text {rd }}$ EPSS/ $27^{\text {th }}$ PSHS Joint Subcommittee meeting, Monterey, CA, 2012. 